\title{
Oxford Textbook of Anesthesia e-book
}

\author{
Jonathan G. Hardman, Philip M. Hopkins, Michel M.R.F Struys (Editors). Oxford \\ University Press - United Kingdom, 2017, 1680 pages. eISBN: 9780191833243, \\ ISBN: 9780199642045. Online access: https://oxfordmedicine.com/view/10.1093/med/ \\ 9780199642045.001.0001/med-9780199642045?rskey=IISLJf\&result=1
}

\author{
Is'haq Al Aamri, BHSc, MD, FRCPC
}

Received: 23 January 2020/Revised: 23 January 2020/Accepted: 26 January 2020/Published online: 4 February 2020

(C) Canadian Anesthesiologists' Society 2020

The Oxford Textbook of Anesthesia e-book is the online version of the first edition (2017) of this book, a twovolume textbook belonging to the Oxford Textbook series. It became available in April 2017 with annual updates and can only be accessed by individuals, groups, or institutions by subscription. The editors include Dr. J.G. Hardman, Professor and Consultant Anaesthetist, Anaesthetics, and Critical Care, University of Nottingham (UK); Dr. P.M. Hopkins, Professor of Anaesthesia and Honorary Consultant Anaesthetist, St James's University Hospital (UK); and Dr. M.M.R.F. Struys, Professor and Chair, Department of Anesthesiology, University of Groningen (The Netherlands) and Professor in Anaesthesia, Ghent University (Belgium). This e-book is multi-authored, with most contributors hailing from the United Kingdom and Europe, with a smattering of contributions from Canada, the United States, New Zealand, Australia, Japan, China, Hong Kong, and Singapore. A comprehensive anesthesia reference text that includes a wide range of anesthesia subspecialties, it targets anesthesia trainees and experienced staff alike.

The textbook is divided into 13 parts with 91 chapters. The first 47 chapters (volume 1) review the basic science and fundamental principles of anesthesia, and the remaining 44 chapters (volume 2) focus on the clinical aspects of anesthesia care. Part 1 (11 chapters) covers the physiological principles of anesthesia, including those focusing on cardiac, vascular, respiratory, gastrointestinal, kidney, acid-base, neuromuscular, neuroendocrine, pain,

I. Al Aamri, BHSc, MD, FRCPC ( $ه)$

McGill University Health Center, Montreal, QC, Canada

e-mail: ishaq.alaamri@mail.mcgill.ca

National Heart Center, Muscat, Oman immune system, and blood physiological concerns. Part 2 (11 chapters) covers applied pharmacology, including drug distribution and elimination; mechanisms and determinants of anesthetic drug actions; inhaled, intravenous, and local anesthetics (each covered in its own chapter); analgesics; cardiovascular drugs; intravenous fluids; neuromuscular blockade and reversal; drug interaction in anesthetic practice; and adverse drug reactions in anesthesia.

Part 3 (four chapters) reviews relevant principles of physics, equipment, measurement and monitoring systems, and assessment of the components of anesthesia (hypnosis/ amnesia, immobility, analgesia). The discussion on anesthesia equipment covers the anesthesia workstation, airway equipment, ventilators, and breathing systems. Part 4 (four chapters) covers some of the important skills the anesthesiologist should acquire along one's career pathway to understand clinical research and the use the evidence produced to deliver safe anesthesia. It reviews mathematical models and anesthesia, presentation and handling of date, descriptive and inferential statistics, evidence-based medicine, and clinical trial design in anesthesia.

Part 5 (nine chapters) covers the history of anesthesia, the role and responsibilities of the anesthesiologist, ethics, patient consent, the benefits and risks in anesthesia practice, informatics and technology for anesthesia, human factors and simulation in anesthetic practice, safety and quality assurance, teaching and research in anesthesia, and anesthesia outcomes (long term). Part 6 (eight chapters) reviews preoperative assessment and optimization, choice and interpretation of preoperative investigation, management of anesthesia, intraoperative monitoring, hazards of anesthetic practice, postoperative 
analgesia and acute pain management, and postoperative and postanesthesia complications.

Part 7 (four chapters) covers important procedures, techniques, and therapies, including the importance of the airway in anesthetic practice, vascular access, conscious sedation, and blood conservation and transfusion in anesthesia. Part 8 (four chapters) covers regional anesthesia topics, including its general principles and regional anesthesia of the trunk and upper and lower limbs.

Part 9 (three chapters) includes the conduct of anesthesia according to surgical specialty. It covers anesthesia for cardiac, thoracic, vascular, ophthalmic, ear-nose-throat, dental and faciomaxillary, abdominal, orthopedic, and trauma cases; anesthesia for transplantation, day-stay surgery, plastic surgery, and burns; for obstetric anesthesia and analgesia; anesthesia for urological surgery and for robotic urological and gynecological surgery.

Part 10 (seven chapters) covers pediatric anesthesia, including the related anatomy, physiology, and pharmacology at the pediatric level; anesthesia for neonates, infants, and older children; procedural sedation in children; acute pediatric pain management; resuscitation, stabilization, and transfer of sick and injured children; and the ethics, consent, and safeguarding required for pediatric anesthesia.

Part 11 (three chapters) reviews anesthesia outside the operating room. It covers pre-hospital care for the anesthetist, anesthesia for medical imaging and bronchoscopic procedures, and military anesthesia. Part 12 (nine chapters) covers anesthesia in regard to concurrent diseases. It thus reviews anesthesia in the presence of cardiac, hepatic, renal, endocrine, and pulmonary diseases; musculoskeletal, hematological, and coagulation disorders; central nervous system pathologies; and in the elderly and patients who are obese. Part 13 (four chapters) focuses on critical care and anesthesia, including preoperative optimization of high-risk patients, acute illness during the postoperative period, transport of critically ill patients, and advanced life support.

The Oxford Textbook of Anesthesia e-book is a comprehensive, wide-ranging, reference textbook that can be accessed in the operating room using "smart" devices or computers. The format allows users to efficiently approach topics of interest, even providing an option to download each chapter in PDF form. The text in each chapter is well organized with cross-linking, arrows for easy text navigation (e.g., "next", "previous", "top"), coloured sub-headings, diagrams, tables, and illustrations. There is also an option to expand and even download the figures.

However, I found it somewhat difficult to navigate within each chapter as no outline is provided. The reader must survey the entire chapter to learn its content. In addition, the text is rather dense and presented in a monochromatic format, making it somewhat tiring to the eyes. These shortcomings could be easily remedied by inserting an outline at the beginning of each chapter that cross-links to the text, key points, and overview. Liberal use of colored text to distinguish subheadings would also help break the monotony. With regard to improving the content, the information concerning the anesthetic machines and vaporizers is a bit wanting - a shortcoming that should be addressed as this topic is essential for both trainees and practicing anesthetists. Finally, the review of regional anesthesia techniques in pediatric patients (children and infants) does not meet the same standards as that provided for adults. In addition, it would benefit from the inclusion of graphs, tables, and figures (perhaps even including ultrasonography scans).

In conclusion, the Oxford Textbook of Anesthesia e-book gives trainees and practicing anesthesiologists access to comprehensive, up-to-date information that covers the realms of basic science and the clinical foundations of anesthesia practice. It covers important topics that are generally not easy to find in a single reference, such as transport of critically ill patients, preoperative optimization of high-risk patients, evidence-based medicine and research in anesthesia, and outcomes after anesthesia. Of note, although most of the authors are from the United Kingdom and Europe, an attempt was made to "globalize" the textbook - such as providing the North American drug formulary counterparts in brackets (e.g., pethidine [meperidine], paracetamol [acetaminophen]) and providing recommendations from non-European societies (e.g., American Heart Association, American Society of Anesthesiologists). I thoroughly enjoyed perusing this ebook and believe that it would be an invaluable addition to any department's electronic library.

\section{Conflicts of interest None.}

\section{Funding statement None.}

Editorial responsibility This submission was handled by Dr. Hilary P. Grocott, Editor-in-Chief, Canadian Journal of Anesthesia.

Publisher's Note Springer Nature remains neutral with regard to jurisdictional claims in published maps and institutional affiliations. 